\title{
An Investigation into the Sinkage of a Ship at the Transcritical Speed in Shallow Water
}

\author{
by Hajime Maruo, Member* Toshiichi Tachibana, Overseas Member**
}

\begin{abstract}
Summary
The bodily sinkage of a ship going in shallow water is analysed by means of the analogy with the transonic aerodynamics. The OGSA method is employed to solve the basic equation in such a way that the nonlinear characteristic is preserved. The numerical work is confined in the subcritical speed range where a continuous solution exists. Three kinds of Wigley's parabolic models with different beam to length ratios are employed for numerical examples. Computed results for the sinkage show fairly good agreement with the measurement in the towing tank, and the feasibility of the method as the practical assessment of the sinkage in shallow water seems to be quite promising.
\end{abstract}

\section{Introduction}

The hydrodynamics of a ship moving in shallow water appeared as early as in the famous paper of Michell (1898) ${ }^{1)}$ and was discussed later by Joukovski $(1903)^{2)}$ who derived independently a similar formulation to that of Michell. Their theories are based on the linearization of the originally nonlinear equation by the assumption of a thin ship. Tuck (1966) $)^{3)}$ has reproduced Michell's linearized theory by a rigorous application of the slender body perturbation and the matched asymptotic expansion and has shown numerical examples for sinkage of the ship. However several experiments such as those by Izubuchi (1937)4) indicates that some irregular phenomena take place in the flow around the hull when the advance velocity approaches the speed of solitary wave at that water depth, and the linearized theory fails to give a right description of actual phenomena. Kreitner $(1934)^{5)}$ has applied the hydraulic theory in one dimension for the open channel to the ship moving in a canal, by which the width of water surface as well as the depth is restricted. It is revealed that the equation for the flow velocity alongside the hull ceases to have a rational solution at a certain speed range around the critical

* Faculty of Engineering, Yokohama National University

** Naval Engineering Division, São Paulo State Technological Research Institute and São Paulo University, Brazil speed. Kreitner's theoretical prediction has been confirmed by extensive model experiments by Kinoshita $(1946,1955)^{6}$. Maruo $(1948,1952)^{\text {?) }}$ has pointed out that the irregular phenomena at the transcritical speed can take place not only in the canal of restricted width but also in the infinite water area of finite depth. He has suggested that the flow field around the hull at the transcritical speed can be analysed by a nonlinear differential equation which appears in the problem of the transonic flow of compressible fluid in two dimensions and has shown the nonexistence of the steady continuous solution near the critical speed by illustrating examples of simple body configurations. Feldman and Lea $(1972)^{8)}$ applied the slender body theory and matched asymptotic expansion and derived a nonlinear differential equation which was quite similar to the transonic aerodynamics. The method of solution employed was analoguous to the quasi-linear approximation developed by Hosokawa $(1960)^{9}$.

The motivation of the present investigation is the practical need for prediction methods of sinkage of a ship on the voyage in Brasilian inland. water. It has been disclosed that existing methods are unable to give predictions with a satisfactory accuracy. In the present work, the method developed by Oswatitsch $(1950)^{10)}$, Gullstrand $(1951)^{11)}$, Spreiter and Alksne $(1958)^{12}$, which is called OGSA method is employed to solve the governing equation. This method enables the solution to preserve the nonlinear characteristic of the basic equation. Numerical 
examples are given to hull forms of parabolic water line, and model tests are conducted for the purpose of comparison with theoretical computations.

\section{Basic equation for shallow water}

Consider a ship being fixed in a uniform flow with velocity $U$ of constant depth $h_{0}$, and assume an irrotational motion of an inviscid incompressible fluid. Take the origin of cartesian coordinates on the bottom of water, with the $x$ aixs in the direction of the uniform flow and the $z$ axis vertically upwards. The disturbed free surface is expressed by $z=h$. Governing equations of the fluid motion are continuity equation

$$
u_{x}+v_{y}+w_{z}=0
$$

kinematical free surface condition

$$
u h_{x}+v h_{y}-w=0 \text { at } z=h
$$

dynamical free surface condition

$$
\frac{1}{2}\left(u^{2}+v^{2}+w^{2}\right)+g h=\frac{1}{2} U^{2}+g h_{0} \quad \text { at } \quad z=h
$$

condition at the bottom

$$
w=0 \text { at } z=0
$$

hull surface condition

$$
v=u F_{x}(x, z)+w F_{z}(x, z) \text { at } y=F(x, z)
$$

Now we assume that the water depth is sufficiently small with respect to the characteristic length, the ship length $L$ say. Then one can write

$$
\begin{aligned}
w)_{z=h} & \left.=\int_{0}^{h} \frac{\partial w}{\partial z} d z=h \frac{\partial w}{\partial z}\right)_{z=h}+O\left(h^{2}\right) \\
& \approx-h\left(\frac{\partial u}{\partial x}+\frac{\partial v}{\partial y}\right)_{z=h}
\end{aligned}
$$

by omitting higher order terms with respect to $h$. If we introduce the above relation in eq. (2), we have

$$
u h_{x}+v h_{y}+h\left(u_{x}+v_{y}\right)=0
$$

Combining with Eq. (3) and omitting higher order terms, we obtain

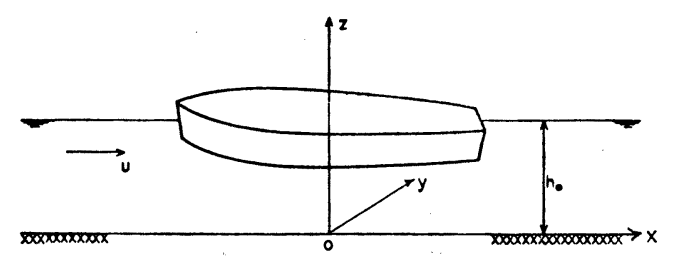

Fig. 1 Coordinate system

$$
\begin{aligned}
\frac{\partial u}{\partial x}+\frac{\partial v}{\partial y}= & \frac{1}{g h}\left(u^{2} \frac{\partial u}{\partial x}+u v \frac{\partial v}{\partial x}\right. \\
& \left.+u v \frac{\partial u}{\partial y}+v^{2} \frac{\partial v}{\partial y}\right) \text { at } z=h
\end{aligned}
$$

Now the velocity potential is introduced because of the irrotational motion.

$$
u=\Phi_{x}, \quad v=\Phi_{y}
$$

Then the basic equation becomes

$$
\begin{aligned}
& \left(1-\frac{\Phi_{x^{2}}}{g h}\right) \Phi_{x x}+\left(1-\frac{\Phi_{y^{2}}}{g h}\right) \Phi_{y y} \\
& -2 \frac{\Phi_{x} \Phi_{y}}{g h} \Phi_{x y}=0
\end{aligned}
$$

If we put

$$
1-\frac{\Phi_{x}^{2}}{g h}=A, \quad \frac{2 \Phi_{x} \Phi_{y}}{g h}=B, \quad 1-\frac{\Phi_{y}^{2}}{g h}=C
$$

we have the equation such as

$$
A \Phi_{x x}-B \Phi_{x y}+C \Phi_{y y}=0
$$

Let us defined $q$ for the fluid velocity, $F_{h}=$ $q / \sqrt{g h}$ for the local depth Froude number and

$$
\Delta=B^{2}-4 A C=4\left(F_{h}^{2}-1\right)
$$

There are three types of flow.

a) $F_{h}<1$ subcritical flow (elliptic equation)

b) $F_{h}=1$ critical flow (parabolic equation)

c) $F_{h}>1$ supercritical flow (hyperbolic equation)

At the transcritical speed, the above three types coexist in the flow field. At the place where the flow changes from supercritical to subcritical, discontinuity of the solution is liable to appear and the shock or the hydraulic jump takes place. The equation (9) is similar to the equation for two-dimensional flow of a compressible fluid, if we replace $\sqrt{g h}$ by the sonic speed $c$, and the depth Froude number corresponds to the Mach number.

Now let us define the perturbation potential $\phi$ such as

$$
U \phi=\Phi-U x
$$

Then fluid velocities are expressed by

$$
\left.\begin{array}{l}
u=U\left(1+\phi_{x}\right)=U(1+\bar{u}) \\
v=U \phi_{y}=U \bar{v}
\end{array}\right\}
$$

There is a relation from (3) such as

$$
g h=g h_{0}-\frac{1}{2} U^{2}\left(2 \bar{u}+\bar{u}^{2}+\bar{v}^{2}\right)
$$

Then eq. (9) gives

$$
\begin{aligned}
\left(1-F_{0}^{2}\right) \phi_{x x}+\phi_{y y}= & F_{0}^{2}\left(3 \phi_{x} \phi_{x x}\right. \\
& \left.+\phi_{x} \phi_{y y}+2 \phi_{y} \phi_{x y}\right)
\end{aligned}
$$


where $F_{0}=U / \sqrt{g h_{0}}$, the depth Froude number of the base flow. If we put

$$
\beta^{2}=1-F_{0}^{2}
$$

$\beta$ is a small fraction at the transcritical speed. Now we apply the Prandtl-Glauert transformation

$$
x=\bar{x}, \quad y=\bar{y} / \beta
$$

and put

$$
\phi=\phi^{*} \beta^{2} /\left(1-\beta^{2}\right)
$$

Then eq. (15) can be written as

$$
\begin{aligned}
& \phi_{\bar{x} \bar{x}} *+\phi_{\bar{y} \bar{y}}{ }^{*}=3 \phi_{\bar{x}} * \phi_{\bar{x} x}{ }^{*}+\beta^{2} \phi_{\bar{x}} * \phi_{\bar{y} \bar{y}} * \\
& +2 \beta^{2} \phi_{y}^{*} \phi_{x}^{-*}
\end{aligned}
$$

Thus the second and third terms on the right hand side can be omitted for small $\beta$. Then we have as the basic equation

$$
\phi_{x \bar{x}} *+\phi_{\bar{y}}^{-*}=3 \frac{\partial}{\partial \bar{x}}\left(\frac{\phi_{x}^{*} * 2}{2}\right)
$$

or, in reference to the original coordinate system,

$$
\beta^{2} \phi_{x x}+\phi_{y y}=K \frac{\partial}{\partial x}\left(\frac{\bar{u}^{2}}{2}\right)
$$

where $K=3 F_{0}^{2}$

\section{The method of solution}

The equation (20) is still nonlinear and its solution is difficult to find. Now let us employ a method which is analogous to what is called OGSA method for transonic flow around a slender body. At the transonic speed, discontinuity in the velocity field can appear, which is the shock wave in the compressible fluid. The analogous phenomenon in shallow water flow is the hydraulic jump. The basic assumption is the weakness of the shock and the irrotationality is preserved behind the shock line. Assuming

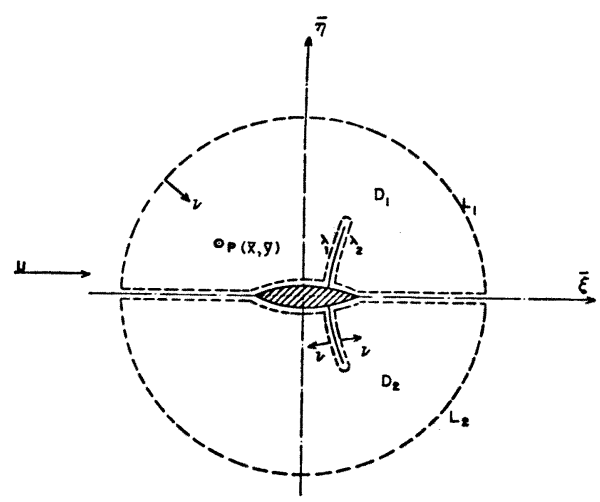

Fig. 2 Integration field for Green's theorem shock lines on both sides of the ship, we apply Green's theorem in the domain $D_{1}$ surrounded by a contour $L_{1}$ in $\bar{\xi}, \bar{\eta}$ plane as shown in Fig. 2.

$$
\begin{aligned}
& \iint_{D_{1}}\left(\phi \nabla^{2} \psi-\psi \nabla^{2} \phi\right) d S \\
& \quad=-\int_{L_{1}}\left(\phi \frac{\partial \psi}{\partial \nu}-\psi \frac{\partial \phi}{\partial \nu}\right) d s
\end{aligned}
$$

where $\psi$ is the Green function defined by

$$
\psi=\frac{1}{2 \pi \beta} \ln \left[(\bar{x}-\bar{\xi})^{2}+(\bar{y}-\bar{\eta})^{2}\right]^{1 / 2}
$$

$v$ stands for the normal directing inward to the contour, and the Laplacian $\nabla^{2}$ is with respect to $\bar{\xi}$ and $\bar{\eta}$. Writing the result in reference to the original coordinate system, we have

$$
\begin{aligned}
\phi(x, y)= & -\int_{-\infty}^{\infty}\left(\psi \phi_{\nu}-\phi \psi_{\nu}\right) d s \\
& -\int_{\lambda_{1}}\left(\psi \phi_{\nu}-\phi \psi_{\nu}\right) d s \\
& -\int_{\lambda_{2}}\left(\psi \phi_{\nu}-\phi \psi_{\nu}\right) d s \\
& -\iint_{D,} K \psi \frac{\partial}{d \xi}\left(\frac{\bar{u}^{2}}{2}\right)_{x=\xi} d S
\end{aligned}
$$

where

$$
\frac{\partial}{\partial \nu}=\beta^{2} n_{x} \frac{\partial}{\partial x}+n_{y} \frac{\partial}{\partial y}
$$

There are integrals along the shock line, with $\lambda_{1}$ for upstream side and $\lambda_{2}$ for downstream side. Integrating the last term by parts, we obtain

$$
\begin{aligned}
\phi(x, y)= & -\int_{-\infty}^{\infty}\left[\psi\left(\phi_{\nu}-K \frac{\bar{u}^{2}}{2} n_{x}\right)-\phi \psi_{\nu}\right] d s \\
& -\int_{\lambda_{1}}\left[\psi\left(\phi_{\nu}-K \frac{\bar{u}^{2}}{2} n_{x}\right)-\phi \psi_{\nu}\right] d s \\
& -\int_{\lambda_{2}}\left[\psi\left(\phi_{\nu}-K \frac{\bar{u}^{2}}{2} n_{x}\right)-\phi \psi_{\nu}\right] d s \\
& +K \iint_{D_{1}} \frac{\bar{u}^{2}}{2} \psi_{\xi} d S
\end{aligned}
$$

Then the integral along the shock line becomes

$$
\begin{aligned}
I_{s}= & -\int_{\lambda_{1}}\left[\psi\left(\phi_{\nu}-\frac{K}{2} \bar{u}^{2} n_{x}\right)-\phi \psi_{\nu}\right] d s \\
& -\int_{\lambda_{2}}\left[\psi\left(\phi_{\nu}-\frac{K}{2} \bar{u}^{2} n_{x}\right)-\phi \psi_{\nu}\right] d s
\end{aligned}
$$

Assuming the irrotational motion for which $\phi$ is continuous across the shock line, we can write

$$
\begin{aligned}
I_{s}= & \int\left\{\psi \left[\left(\phi_{\nu}-\frac{K}{2} \bar{u}^{2} n_{x}\right)_{\lambda_{1}}\right.\right. \\
& \left.\left.+\left(\phi_{\nu}-\frac{K}{2} \bar{u}^{2} n_{x}\right)_{\lambda_{2}}\right]\right\} d s=\int \psi S_{1} d s
\end{aligned}
$$

where 


$$
\begin{aligned}
S_{1}= & \left(\phi_{x} \beta^{2} n_{x}+\phi_{y} n_{y}-\frac{K}{2} \bar{u}^{2} n_{x}\right)_{\lambda_{1}} \\
& +\left(\phi_{x} \beta^{2} n_{x}+\phi_{y} n_{y}-\frac{K}{2} \bar{u}^{2} n_{x}\right)_{\lambda_{2}}
\end{aligned}
$$

Now let us consider the jump condition on the discontinuous surface. If the conservation law is expressed by the quasi-linear system

$$
p_{x}(x, y, u, v)+q_{v}(x, y, u, v)+n(x, y, u, v)=0
$$

the jump condition associated with the above equation becomes

$$
\gamma_{x}\langle p\rangle+\gamma_{y}\langle q\rangle=0
$$

where $\gamma_{x}, \gamma_{y}$ are direction cosines of the normal to the shock line, and $\langle p\rangle,\langle q\rangle$ are jump of $p$ and $q$ across the shock line respectively. ${ }^{13)}$ Since our case is

$$
\begin{aligned}
& p=\beta^{2} \bar{u}-\frac{K}{2} \bar{u}^{2} \\
& q=v
\end{aligned}
$$

from eq. (20), the jump condition becomes

$$
\left\langle\beta^{2} \bar{u}-\frac{K}{2} \bar{u}^{2}\right\rangle n_{x}+\langle v\rangle n_{y}=0
$$

Therefore we have

$$
S_{1}=0
$$

Thus the integrals along the shock line vanishes and the velocity potential can be expressed by

$$
\begin{aligned}
\phi(x, y)= & -\int_{-\infty}^{\infty}\left(\psi \phi_{\nu}-\phi \psi_{\nu}\right) d s \\
& +K \iint_{D_{1}} \frac{\bar{u}^{2}}{2} \psi_{\xi} d S
\end{aligned}
$$

A similar application of Green's theorem to the domain $D_{2}$ encompassed by the closed contour $L_{2}$ in the lower half plane of Fig. 2 leads to

$$
0=-\int_{-\infty}^{\infty}\left(\psi \phi_{\nu}-\phi \psi_{\nu}\right) d s+K \iint_{D_{2}} \frac{\bar{u}^{2}}{2} \psi_{\xi} d S
$$

Now we introduce the assumption of the slender body. Then the integrals along $L_{1}$ and $L_{2}$ are reduced to those along the $x$ axis, and there are relations

$$
\begin{aligned}
& \left.\left.\frac{\partial \psi}{\partial \nu}\right)_{L_{1}}=-\frac{\partial \psi}{\partial \nu}\right)_{L_{2}} \quad \text { on the } x \text { axis } \\
& \begin{array}{ll}
\left.\left.\left.\frac{\partial \phi}{\partial \nu}\right)_{L_{1}}=\frac{\partial \phi}{\partial \nu}\right)_{L_{2}}=\frac{\partial \phi}{\partial \eta}\right)_{\eta=0}=F^{\prime}(\xi) \\
\text { for }-L / 2 \leq x \leq L / 2
\end{array} \\
& \begin{array}{ll}
\left.\frac{\partial \phi}{\partial \eta}\right)_{\eta=0}=0 & \text { for } \quad|x|>L / 2
\end{array}
\end{aligned}
$$

where $y= \pm F(x)$ is the equation of the waterline.
Summing up (30) and (31) and applying the above relations, we obtain

$$
\begin{aligned}
\phi(x, y)= & -2 \int_{-L / 2}^{L / 2} F^{\prime}(\xi) \psi d \xi \\
& +K \iint_{D_{1}+D_{2}} \frac{\bar{u}^{2}}{2} \psi \xi d \xi d \eta
\end{aligned}
$$

The first term on the right hand side is rewritten by the integration by parts resulting

$$
\phi_{L}=\frac{1}{\pi \beta} \int_{-L / 2}^{L / 2} F(\xi) \frac{x-\xi}{(x-\xi)^{2}+\beta^{2} y^{2}} d \xi
$$

and is identical with the solution of the linearized theory. Differentiating (32) with respect to $x$ and putting

$$
\bar{u}_{L}=\partial \phi L / \partial x
$$

we have

$$
\begin{gathered}
\bar{u}(x, y)=\bar{u}_{L}+\frac{K}{2 \beta^{2}} \frac{\bar{u}^{2}}{2}-\frac{K}{\pi \beta} \iint_{-\infty}^{\infty} \frac{\bar{u}^{2}(\xi, \eta)}{2} \\
\cdot \frac{(x-\xi)^{2}-\beta^{2}(y-\eta)^{2}}{\left[(x-\xi)^{2}+\beta^{2}(y-\eta)^{2}\right]^{2}} d \xi d \eta
\end{gathered}
$$

The second term on the right hand side comes from the integral inside the small circle around the field point $\mathrm{P}$ shown in Fig. 2. The above can be written in the form like

$$
\bar{u}=\bar{u}_{L}+\frac{K}{2 \beta^{2}} \frac{\bar{u}^{2}}{2}-\frac{K}{\pi \beta} I
$$

which is similar to the equation obtained by Oswatitsch. This is a nonlinear integral equation for the velocity $\bar{u}$ and an iterative method is employed to solve it. Oswatitsch proposed an approximation for $\bar{u}$ inside the integral sign such as

$$
\bar{u}(\xi, \eta)=\frac{\vec{u}_{s}(\xi)}{(1+\eta / V)^{2}}
$$

where $\bar{u}_{s}(\xi)$ is the velocity on the surface of the body and $V$ is determined by the irrotational condition for $\bar{u}$ on the $x$ axis, resulting

$$
V=-2 \bar{u}_{s} / F^{\prime \prime}(\xi)
$$

In order to carry out the iteration, we have to choose an appropriate test function for the first approximation of $\bar{u}_{s}(\xi)$. If $\bar{u}_{L}$ and $I$ are assumed to be known functions, (35) becomes a nonlinear algebraic equation of $\bar{u}$. When the uniform flow is sufficiently subcritical, one can begin with the test function for $\bar{u}_{s}$ equal to $\bar{u}_{L}$. The next stage is the substitution of the solution of (35) in $I$ and iteration is finished when $\bar{u}_{s}(\xi, 0)$ coincides with assumed $\bar{u}_{\boldsymbol{s}}$. Spreiter and Alksne have shown that the solution converges after a few iterations.

It shoud be pointed out that eq. (35) can be 
transformed to a form which is independent of $\beta$ or $F_{0}$. If we put

$$
\bar{u}=\frac{\beta^{2}}{K} u^{*}, \quad \bar{u}_{L}=\frac{\beta^{2}}{K} u L^{*}
$$

and

$$
I^{*}=\iint \frac{u^{* 2}}{2} \frac{(\bar{x}-\bar{\xi})^{2}-(\bar{y}-\bar{\eta})^{2}}{\left[(\bar{x}+\bar{\xi})^{2}+(\bar{y}-\bar{\eta})^{2}\right]^{2}} d \overline{\bar{\xi}} d \bar{\eta}
$$

the equation becomes

$$
u^{*}=u L^{*}+\frac{u^{* 2}}{4}-\frac{1}{\pi} I^{*}
$$

Therefore a similar solution is obtained with respect to the coordinate system $\bar{x}, \bar{y}$ with a similarity parameter $\left(1-F_{0}{ }^{2}\right) / 3 F_{0}{ }^{2}$.

\section{Numerical results for sinkage and comparison with experiment}

The vertical force acting on the slender ship is given by

$$
F_{2}=\int p(x) B(x) d x
$$

where $B(x)$ is the breadth of the water plane and

$$
p(x)=-\rho U^{2} \bar{u}(x, 0)
$$

The moment about the $y$ axis is given by

$$
M_{y}=-\int p(x) B(x) x d x
$$

If the bodily sinkage at the midship section is $s$ and the change of trim angle is $\theta$, there are equations of equilibrium such as

$$
\begin{aligned}
& -F_{2}=\rho g \int(s+\theta x) B(x) d x \\
& M_{y}=\rho g \int(s+\theta x) x B(x) d x
\end{aligned}
$$

Then the sinkage and trim are given by

$$
\begin{aligned}
& \frac{s}{L}=F_{N^{2}} \frac{C F-C M \cdot C A}{1-C A \cdot C B} \\
& \frac{\theta}{L}=F_{N^{2}} \frac{C M-C B \cdot C F}{1-C A \cdot C B} \\
& F_{N}=U / \sqrt{g L} .
\end{aligned}
$$

where

$$
\begin{aligned}
& C F=\frac{\int \bar{u}(x, 0) B(x) d x}{\int B(x) d x} \\
& C M=\frac{\int \bar{u}(x, 0) x B(x) d x}{\int x^{2} B(x) d x} \\
& C A=\frac{\int x B(x) d x}{\int B(x) d x}
\end{aligned}
$$

$$
C B=\frac{\int x B(x) d x}{\int x^{2} B(x) d x}
$$

As a numerical example, Wigley's parabolic model is employed. The equation of the hull surface is

$$
y=\frac{B}{2}\left[1-\left(\frac{2 x}{L}\right)^{2}\right]\left[1-\left(\frac{z}{T}\right)^{2}\right]
$$

Three kinds of models are considered, principal dimensions of which are given in Table 1. The present calculation is confined in the subcritical speed range of the base flow where a continuous solution of eq. (35) exists. Because of the symmetric model, the flow velocity is symmetric with respect to the midship section in the speed range of the calculation and there is no trim change consequently. The bodily sinkage is determined by eq. (46). Numerical results for Model 166 is shown in Fig. 3, where the result by Tuck's linearized theory is also illustrated. The effect of the water depth does not appear in the latter, but the nonlinear calculation shows the dependence on the water depth. It is of some interest to observe that the linearized result does not represent an asymptote of the nonlinear result.

Table 1 Principal dimensions of models

\begin{tabular}{|l|l|l|l|}
\hline Iumber of Model & 166 & 165 & 158 \\
\hline Lenght (m) I & 1.500 & 1.500 & 3.048 \\
\hline Breadth (m) B & 0.100 & 0.125 & 0.435 \\
\hline Draught (m) I & 0.100 & 0.100 & 0.189 \\
\hline B/L & 0.067 & 0.083 & 0.143 \\
\hline T/L & 0.067 & 0.067 & 0.062 \\
\hline Displacement (kg) & 17.750 & 18.200 & 130.30 \\
\hline Wetted Surface(m) & 0.436 & 0.488 & 1.633 \\
\hline
\end{tabular}

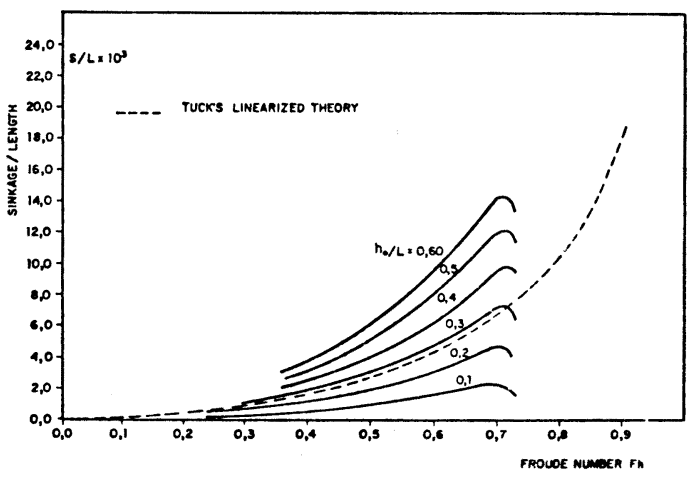

Fig. 3 Computational results for sinkage of model 166 


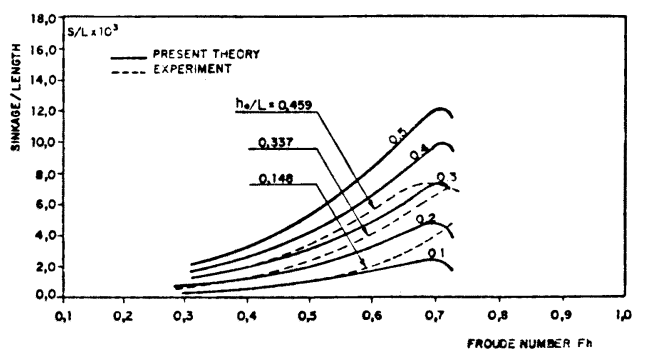

Fig. 4 Sinkage of model 166, comparison of computation and experiment

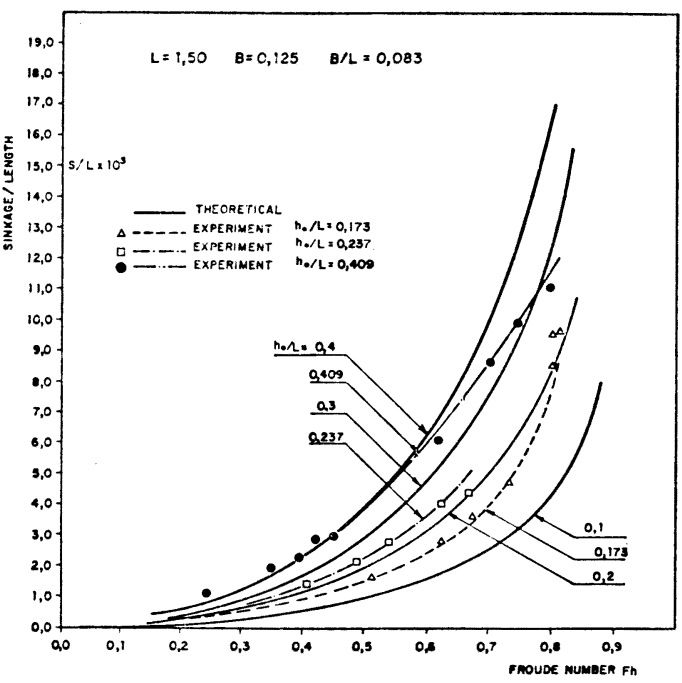

Fig. 5 Sinkage of model 165, comparison of computation and experiment

Model tests are conducted at São Paulo State Technological Research Institute (IPT). The water level is reduced at a part of the towing tank with width 3.5 metre. According to Kinoshita's experiment, the restriction of tank width results a serious effect on the flow pattern at the critical speed. The most remarkable phenomenon is the stagnant wave which appears in front of the model and causes an excessive trim by stern. Since the tank width is not so large in the present experiment, the effect of the restricted width may appear when the model speed comes close to the critical speed. Therefore the comparison of computation and measurement is valid in the speed below that of the inception of stagnant wave. Computed and measured results for three models are shown in Figs. 4, 5 and 6 respectively. Some discrepancy is observed between computation and measurement for the finest model 166, but more satisfactory agreement is achieved by fuller model 165. Model

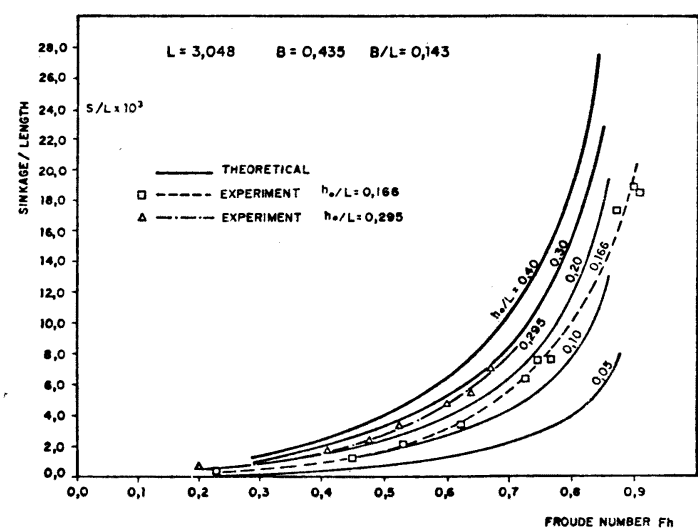

Fig. 6 Sinkage of model 158, comparison of computation and experiment

158 has a proportion of practical hulls. What is interesting is that the best agreement is obtained by the result of the widest model 158. The reason is not clear, but the result is encouraging in view of the feasibility of the present method for the practical application. The droop tendency of the curve of Model 166 near the Froude number 0.7 indicates some irregular behavior of the solution, while it does not appear in the other models. This fact may have some correlation with the poor agreement of the finest model.

\section{Conclusion}

The remarkable sinkage of a ship which takes place when the speed of advance approaches the critical speed in shallow water is analysed by a method which is analoguous to the theory of transonic aerodynamics. The nonlinearity of the basic equation plays an important role in the determination of the fluid motion at the transcritical speed. The linearized theory is not able to predict the sinkage with tolerable accuracy. The result of computation by means of the OGSA method, which preserves the nonlinearity of the basic equation, shows good agreement with measured results, and the application of the present analysis to the assessment of the bodily sinkage in shallow water seems quite promising. At the critical speed, the continuous solution is liable to break down and further investigations are needed for the behavior at the critical speed.

\section{Acknowledgements}

The authors express their thanks to Professor Shin Tamiya of Tsukuba University, whose encouragement and support enabled the junior author to have an opportunity of studying in 
Japan. Their thanks are also to staffs of IPT for assistance in the experimental work.

\section{References}

1) Michell, J. H.: The wave resistance of a ship. Phil. Mag. (5) 45 (1898).

2) Joukovski, N. E.: On the wave of translation. Complete Works 4 ONTI (1937).

3) Tuck, E. O.: Shallow water flows past slender bodies. J.F.M. 26 (1966).

4) Izubuchi, T., Nagasawa, S.: Experimental investigation on the influence of depth of water upon the resistance of ships. (in Japanese) J.S.N.A. 61 (1937).

5) Kreitner, J.: Über den Schiffswiderstand auf beschrenkten Wasser. W.R.H. (1934).

6) Kinoshita, M.: On the restricted-water effect on ship resistance. Society of Naval Architects of Japan Spring Meeting 1946, J.S.N.A. 76 (1954).

7) Maruo, H.: On the shallow water effect. Soc. of Naval Arch. of Japan Autumn
Meeting 1948, J.S.N.A. 84 (1952).

8) Feldman, J. P., Lea, G. K.: Transcritical shallow water flow past slender ships. 9th Symposium on Naval Hydrodynamics (1972).

9) Hosokawa, I.: A refinement of the linearized transonic flow theory. Journal of Physical Society of Japan 15-1 (1960).

10) Oswatitsch, K.: Die Geschwindigkeitsverteilung bei lokalen Uberschallgebieten an flachen Profilen. Z.A.M.M. 30 (1950).

11) Gullstrand, T. R.: The flow over symmetrical aerofoils without incidence in the lower transonic range. Roy. Inst. Technol. Tech. Note AERO 20 (1951).

12) Spreiter, J. R., Alksne, A. Y.: Theoretical prediction of pressure distribution on nonlifting airfoils at high subsonic speeds. NACA Rept. 1217 (1955).

13) Courant, R., Hilbert, D.: Methods of Mathematical Physics Vol. II p. 488. 